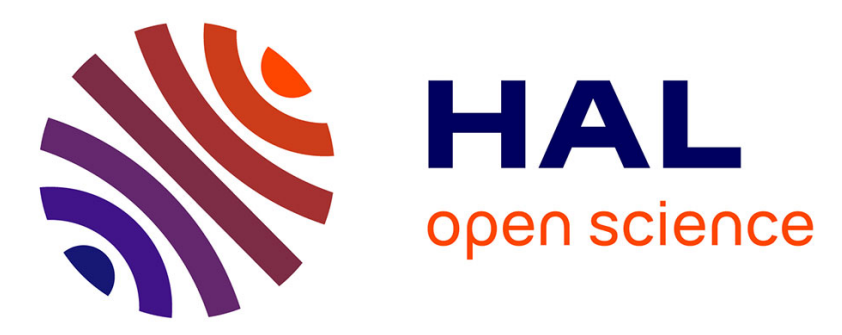

\title{
Weak Stripe Angle Determination by Quantitative x-ray Magnetic Microscopy
}

K Ait Oukaci, D. Lacour, D. Stoeffler, B. Sarpi, F. Montaigne, R. Belkhou, M. Hehn

\section{- To cite this version:}

K Ait Oukaci, D. Lacour, D. Stoeffler, B. Sarpi, F. Montaigne, et al.. Weak Stripe Angle Determination by Quantitative x-ray Magnetic Microscopy. Physical Review Applied, 2020, 14, 10.1103/PhysRevApplied.14.024083 . hal-02928269

\section{HAL Id: hal-02928269 \\ https://hal.science/hal-02928269}

Submitted on 2 Sep 2020

HAL is a multi-disciplinary open access archive for the deposit and dissemination of scientific research documents, whether they are published or not. The documents may come from teaching and research institutions in France or abroad, or from public or private research centers.
L'archive ouverte pluridisciplinaire HAL, est destinée au dépôt et à la diffusion de documents scientifiques de niveau recherche, publiés ou non, émanant des établissements d'enseignement et de recherche français ou étrangers, des laboratoires publics ou privés. 


\title{
Weak Stripe Angle Determination by Quantitative x-ray Magnetic Microscopy
}

\author{
K. Ait Oukaci®, ${ }^{1,2}$ D. Lacour $\odot,{ }^{1}$ D. Stoeffler, ${ }^{3}$ B. Sarpi, ${ }^{2}$ F. Montaigne $\odot,{ }^{1}$ R. Belkhou, ${ }^{2}$ and \\ M. Hehn ${ }^{1, *}$ \\ ${ }^{1}$ Institut Jean Lamour, CNRS - Université de Lorraine, 54011 Nancy, France \\ ${ }^{2}$ SOLEIL Synchrotron, L'Orme des Merisiers, Saint Aubin - BP 48, 91192 Gif-sur-Yvette, France \\ ${ }^{3}$ IPCMS UMR 7504 CNRS, Université de Strasbourg, 23 Rue du Loess, BP 43, 67034 Strasbourg Cedex 2, France
}

(Received 8 March 2020; revised 17 July 2020; accepted 4 August 2020; published 27 August 2020)

\begin{abstract}
High-resolution scanning transmission x-ray microscopy (STXM) can provide quantitative access to local magnetization canting angles relative to the surface. We use this cutting-edge microscopy technique to probe the weak stripe magnetic texture hosted by a 180 -nm-thick $\mathrm{Co}_{40} \mathrm{Fe}_{40} \mathrm{~B}_{20}$ layer. We report a full set of measurements of the weak stripe canting angle, as well as a method that uses only a single-impingingdirection $\mathrm{x}$-ray beam to extract the angle. This method also allows the spatial profile to be extracted with $30 \mathrm{~nm}$ resolution, and the canting angle versus applied field can be measured. Combining these information, the macroscopic magnetization versus applied field loop on a complex spin texture can be reconstructed in an unprecedented way and shows undoubtedly that flux closure domains exist. These results show that the presence of flux closure domains, at the surface of such a thick film, is difficult to demonstrate by means of the STXM technique. Beyond the characterization of the weak stripe angle, this quantitative x-ray magnetic microscopy can be used to study the local textures hosted in all materials exhibiting some circular x-ray magnetic dichroism.
\end{abstract}

DOI: 10.1103/PhysRevApplied.14.024083

\section{INTRODUCTION}

The growing use of magnetic materials in advanced technologies [spin transfer torque magnetic random access memory (STTMRAM), hard drive read heads, magnetic field sensors] is accompanied by a growing need for local magnetic texture characterization. During the last decades, a large variety of textures have been stabilized by changing the intrinsic properties of the magnetic materials themselves, changing their interfaces or shapes [1]. One well-known and observed case is the development of stripe domains in thin magnetic films with a perpendicular anisotropy axis of value $K$, which promotes magnetization perpendicular to the film plane. Competition with the thin-film shape anisotropy, equal to $\left(\mu_{0} / 2\right) M_{S}^{2}\left(M_{S}\right.$ is the material magnetization at saturation), induces in-plane magnetization when a film is typically several nanometers thick. However, above a critical thickness [1-3], the magnetization starts to deviate from the film plane by stabilizing magnetic stripes, a wavy structure in which a small component of perpendicular magnetization develops (see Fig. 1). It alternates between up and down across the stripes to decrease the demagnetization energy. So, the magnetization makes an angle $\theta(y)(=\pi / 2-\varphi$ in Fig. 1) with respect to the film plane with a maximum angle $\pm \theta^{\max }$

*michel.hehn@univ-lorraine.fr at the center of a stripe. $\theta^{\max }$ can vary between zero and $\pi / 2$. Such a magnetic configuration is often called a "weak stripe" (WS). As the layer thickness is increased, the situation where $\theta^{\max }=\pi / 2$ is encountered and is assigned to the "strong-stripe" (SS) phase. While the presence of stripes can be unveiled by a large variety of imaging methods with adequate resolution, determining the precise stripe structure [i.e., $\theta(y)$ and $\theta^{\max }$ ] is not possible. Therefore, global magnetometry techniques are used, since both phase (WS and SS) have different hysteretic responses [1]. From those macroscopic measurements, determining $\theta(y)$ and $\theta^{\max }$ remains challenging due to both the presence of closure domains and assumptions on the magnetization distribution. In 2015, Blanco-Roldán et al. obtained $\theta^{\max }$ for an amorphous $\mathrm{NdCo}_{5}$ thin film hosting a weak stripe domain structure [4]. To do so, they acquired a series of transmission $\mathrm{x}$-ray microscopy images (TXM), varying the impinging angle of the x-ray beam over a range of $100^{\circ}$. Here, we extend their work to measure the magnetization angle distribution using a single impinging angle, taking advantage of x-ray-beam polarization. The method we use saves acquisition time and extends the use of TXM to scanning TXM (STXM), in which the accessible range of impinging angles is limited by the use of zone plates, and goniometric membrane holders are rarely used. Furthermore, we are able to measure the magnetization canting angle versus in-plane applied field and to extract the spatial 


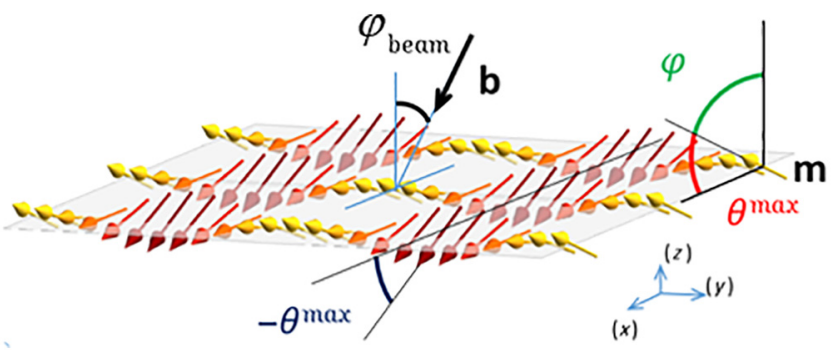

FIG. 1. Sketch of the weak stripe texture and a definition of the angle with beam $\mathbf{b}$ impinging the sample at $\varphi_{\text {beam }}$ to the sample surface normal. Colors of the arrows are linked to $\theta$ values (yellow corresponds to $\theta^{\max }$, brown to $-\theta^{\max }$ ).

profile of magnetization with $30 \mathrm{~nm}$ resolution. This allows us to reconstruct the total magnetization versus applied field loop and compare it to macroscopic measurements. As a result and in contrast to previous reports [4], we also highlight the limits of STXM, which cannot show the existence of flux closure domains.

A large variety of materials are shown to develop WSs and the conclusions of our study could be extended to those materials such as Co-Gd [5], Fe-Pt [6], (Fe, Co)AlON [7], Fe-Zr-N [8], $\mathrm{Fe}_{80} \mathrm{Ga}_{20}$ [9], (Fe, $\left.\mathrm{Si}\right) \mathrm{B}[10,11]$, and $\mathrm{Fe}-\mathrm{Ni}$ [12-14]. Depending on the material's properties, film composition [12-14], and deposition conditions $[2,3,15,16]$, the critical thickness leading to WS appearance varies from $20 \mathrm{~nm}$ for epitaxial Co [17] and $100 \mathrm{~nm}$ for $(\mathrm{Fe}, \mathrm{Ta}) \mathrm{C}$ [18] to $355 \mathrm{~nm}$ for $\mathrm{Ni}_{82} \mathrm{Fe}_{18}$ [14]. WSs are also observed in amorphous $(\mathrm{Co}, \mathrm{Fe}) \mathrm{B}$ films $[16,19,20]$. We choose this last material, since it has many attractive properties (low damping, high magnetic response in very low magnetic field, high magnetoresistance tunnel junctions), which make it a very good basis for application in spintronic-based devices (STTMRAM, hard drive read heads, and low- and highfrequency magnetic sensors [21]). This periodically modulated magnetic texture can also be used as a medium of choice for reprogrammable magnetic devices, in which spin waves are channeled into the magnetic domains [22] or pinning centers for magnetic vortices [23]. On the basis of Ref. [19], the thickness of the $\mathrm{Co}_{40} \mathrm{Fe}_{40} \mathrm{~B}_{20}$ layer is fixed to $180 \mathrm{~nm}$ to obtain WS domains.

\section{EXPERIMENTAL DETAILS}

Amorphous thick $\mathrm{Co}_{40} \mathrm{Fe}_{40} \mathrm{~B}_{20}$ thin films are grown in an Alliance Concept UHV sputtering system on a 5-nmthick Ta seed layer deposited both on a thin SiN membrane from Silson Co. for STXM measurements and on a (100) Si substrate for magnetic force microscopy (MFM). The thin films are capped with a 5-nm-thick Pt layer to prevent oxidation. The macroscopic hysteresis cycles are recorded using an ADE vibrating sample magnetometer with the magnetic field applied in plane. Magnetic imaging is performed using both Asylum MFM and the STXM end station of the HERMES beamline (SOLEIL Synchrotron) [24]. Considering the thickness of the $\mathrm{Co}_{40} \mathrm{Fe}_{40} \mathrm{~B}_{20}$ layer, we work with a beam energy tuned to the Co $L_{2}$ edge $(790 \mathrm{eV})$ to avoid saturation effects and nonlinearity of the x-ray absorption spectroscopy; furthermore, the $L_{3}$ edge is totally absorbed for thicker layers. The membrane holder is surrounded by a quadrupole magnet, allowing magnetic fields to be applied either in plane or perpendicular to the film plane with a maximum amplitude of $200 \mathrm{mT}$.

\section{RESULTS}

As a first step and similar to many studies, the stripe domain structure is observed by conventional microscopy, here MFM. The stripe domains, stabilized after in-plane saturation, are very regular without defects within a scan area of $50 \times 50 \mu \mathrm{m}^{2}$ (Fig. 2).

This contrasts with the report on $(\mathrm{Co}, \mathrm{Fe}) \mathrm{B}$ [19] and gives credit to all WS domain image processing that is reported here. Indeed, as shown in Fig. 2, in all experiments, the stripe domain pattern is prepared and oriented parallel to the plane of incidence of the x-ray beam that impinges on the surface. The in-plane hysteresis cycle is characteristic of a WS domain structure, even if the MFM image cannot give any information on the canting angle. These stripes keep their directivity for all applied fields, except in the region close to $H_{c}$, which is the in-plane coercive field of the film equal to $3 \mathrm{mT}$.

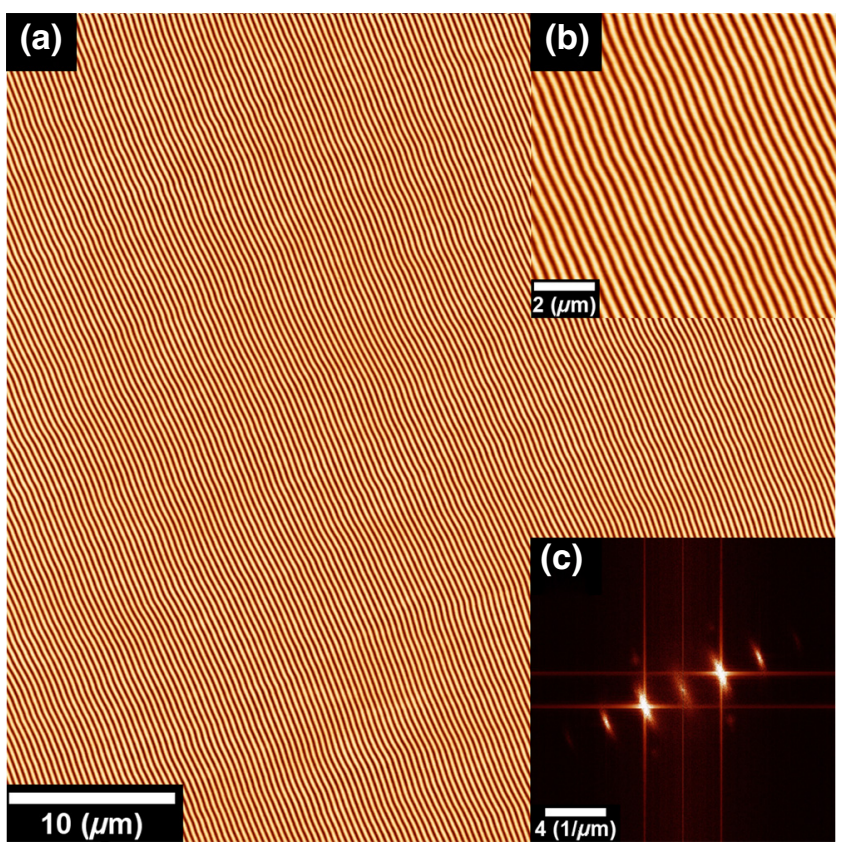

FIG. 2. (a) $50 \times 50 \mu \mathrm{m}^{2}$ MFM image recorded at remanence after a saturation at 800 Oe. (b) $10 \times 10 \mu \mathrm{m}^{2} \mathrm{MFM}$ image recorded under the same conditions. (c) Two-dimensional Fourier transforms of $50 \times 50 \mu \mathrm{m}^{2}$ MFM image that allows the measurement of a stripe period of $315 \mathrm{~nm}$. 
(a)

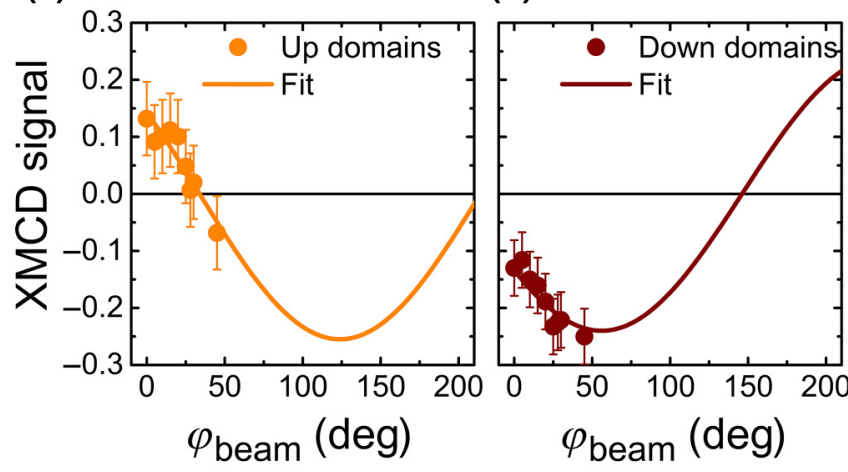

FIG. 3. XMCD signal measured at the center of (a) up domains (domains with angle $\theta^{\max }$ ) and (b) down domains (domains with angle $-\theta^{\max }$ ) as a function of the x-ray-beam impinging angle with respect to the surface. Solid lines are fits using a cosine variation of XMCD.

As a second step, the $\mathrm{x}$-ray magnetic circular dichroism signal (XMCD), equal to $\left(I^{\mathrm{CL}}-I^{\mathrm{CR}}\right) /\left(I^{\mathrm{CL}}+I^{\mathrm{CR}}\right)$, is measured at the center of both the up domain (domain with maximum canting of $\theta^{\max }$ ) and the down domain (domain with maximum canting of $-\theta^{\max }$ ) as a function of the impinging angle of the x-ray beam, as reported in Figs. 3(a) and $3(\mathrm{~b})$, respectively. $I^{\mathrm{CL}}\left(I^{\mathrm{CR}}\right)$ corresponds to the intensity transmitted when circular left (circular right) polarization of the x-ray beam is used [25]. Starting from zero and increasing $\varphi_{\text {beam }}$, the XMCD signal of the up domain decreases and equals zero when $\varphi_{\text {beam }}=\theta^{\max }$. Since XMCD gives a projection of magnetization along the photon beam direction, angular variations are fitted using $\cos \left[(\pi / 2)-\theta^{\max }+\varphi_{\text {beam }}\right]$ and $\cos \left[(\pi / 2)+\theta^{\max }+\right.$ $\left.\varphi_{\text {beam }}\right]$, the variations expected from the schematic in Fig. 1 if the stripe angles are symmetric with respect to the film plane and $\theta^{\max }$ is the maximum magnetization angle. The best fit gives a value of $\theta^{\max }=33.9^{\circ} \pm 2.8^{\circ}$.

In the third step, we show how to extract the WS angle from a single STXM measurement, in which the x-ray beam is not perpendicular to film plane, by the use of photon light polarization. The experimental geometry is sketched in Fig. 1. As discussed in Ref. [4], the transmitted beam intensity can, in general, be written as

$$
\begin{aligned}
& I^{\mathrm{CL}}=I_{0} e^{-\frac{T}{\cos \left(\varphi_{\text {beam }}\right)} \frac{1}{L}(1-d \cdot \mathbf{b} \cdot \mathbf{m})}, \\
& I^{\mathrm{CR}}=I_{0} e^{-\frac{T}{\cos \left(\varphi_{\text {beam }}\right)} \frac{1}{L}(1+d \cdot \mathbf{b} \cdot \mathbf{m})},
\end{aligned}
$$

where $T$ is the film thickness, $L$ is the x-ray attenuation length, $d$ is the maximum magnitude of the dichroic absorption, and $I_{0}$ is the incident beam intensity through the membrane without the ferromagnetic layer. $\mathbf{b}$ and $\mathbf{m}$ are unit vectors defining the beam and magnetization directions, respectively. From Eq. (1), $d \cdot \mathbf{b} \cdot \mathbf{m}$ can be extracted from the calculation of a contrast function that is related to the XMCD signal as

$$
C_{\varphi}^{\varphi_{\text {beam }}}=\frac{\ln \left(I^{\mathrm{CL}} / I^{\mathrm{CR}}\right)}{\ln \left(I^{\mathrm{CL}} I^{\mathrm{CR}} / I_{0}^{2}\right)}=d \cdot \mathbf{b} \cdot \mathbf{m}=-d \cos \left(\varphi_{\text {beam }}+\varphi\right) .
$$

Simple trigonometric calculations lead to $C_{\varphi}^{\varphi_{\text {beam }}}-$ $C_{\pi-\varphi}^{\varphi_{\text {beam }}}=-2 d \cos \varphi_{\text {beam }} \cos \varphi$ and $C_{\varphi}^{\varphi_{\text {beam }}}+C_{\pi-\varphi}^{\varphi_{\text {beam }}}=-2 d$ $\sin \varphi_{\text {beam }} \sin \varphi . C_{\varphi}^{\varphi \text { beam }}$ is calculated directly from $I^{\mathrm{CL}}, I^{\mathrm{CR}}$, and $I_{0}$. For symmetry reasons, if the magnetic texture is translated by half a period in the $y$ direction perpendicular to the stripes, angle $\varphi$ becomes $\pi-\varphi$. $C_{\pi-\varphi}^{\varphi_{\text {beam }}}$ is calculated from $I^{\mathrm{CL}}, I^{\mathrm{CR}}$, and $I_{0}$ and is translated by half of a stripe period. Consequently, the profile of the weak stripe angle can be extracted by the use of a single impinging beam angle using

$$
\varphi=\arctan \left(\frac{1}{\tan \varphi_{\text {beam }}} \frac{C_{\varphi}^{\varphi_{\text {beam }}}+C_{\pi-\varphi}^{\varphi_{\text {beam }}}}{C_{\varphi}^{\varphi_{\text {beam }}}-C_{\pi-\varphi}^{\varphi_{\text {beam }}}}\right) .
$$

This allows us to compute $\theta(y)=\pi / 2-\varphi$, which is presented in Fig. 4 for $\varphi_{\text {beam }}=30^{\circ}$. A cosine function reproduces accurately the experimental $\theta(y)$ profile (see red line in Fig. 4). These measurements can be made with other values of $\varphi_{\text {beam }}$, except $\varphi_{\text {beam }}=0^{\circ}$ : the incident x-ray beam has to be oblique with respect to the sample surface. A measurement at $\varphi_{\text {beam }}=45^{\circ}$ leads to the same profile and value of $\theta^{\max }$. As a result, a $\theta^{\max }$ value of $34.4^{\circ}$ can be extracted.

In the last step, we measure the variation of $\theta^{\max }$ with inplane applied field $H_{x}$. Since the field direction is always perpendicular to the $\mathrm{x}$-ray photon beam, the field cannot be applied in plane with the sample tilted to $\varphi_{\text {beam }}=30^{\circ}$. Therefore, the images collected as a function of applied field are acquired at $\varphi_{\text {beam }}=0^{\circ}$ for different values of $H_{x}$,

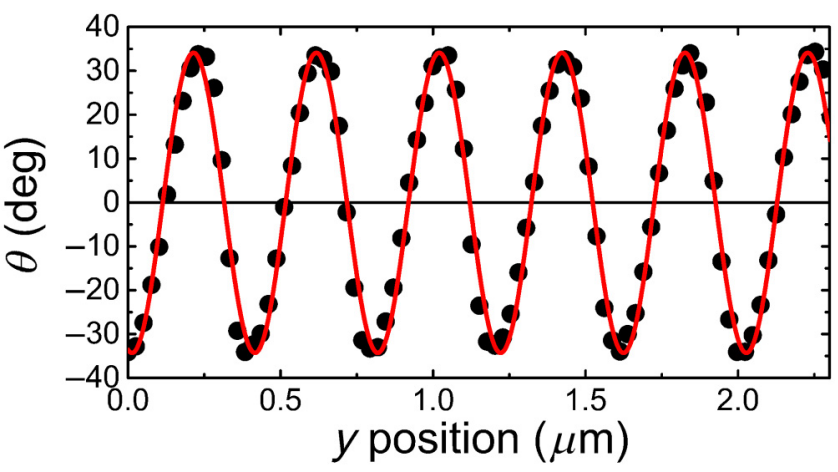

FIG. 4. Measurement of the magnetization angle, $\theta$, versus $y$ position. Data are obtained after averaging the contrasts along the $x$ direction. Red curve is the fit with a cosine variation of the stripe angle with $y$. 
which is the magnetic field applied along $x$. However, in this configuration, Eq. (3) cannot be used, as explained previously. We need to measure conventionally the XMCD at the center of the stripes, either up or down:

$$
\frac{I^{\mathrm{CL}}-I^{\mathrm{CR}}}{I^{\mathrm{CL}}+I^{\mathrm{CR}}}=\tanh \left[\frac{T}{L} d \cos (\varphi)\right] .
$$

To obtain $\theta^{\max }$, it is required to evaluate the product $(T / L) d$. The easiest way would be to measure $\mathrm{XMCD}$ in a saturated state either in plane or out of plane. Since these configurations cannot be accessed, we directly extract $(T / L) d$ from the XCMDs in the center of up and down stripes, since $\theta^{\max }$ is known at zero field. In the center of up domains, $\mathrm{XMCD}_{\text {up }}=$ $\tanh \left\{(T / L)\left[1 / \cos \left(\varphi_{\text {beam }}\right)\right] d \cos \left[(\pi / 2)+\varphi_{\text {beam }}-\theta^{\max }\right]\right\}$, while, in the center of down domains, $\mathrm{XMCD}_{\text {down }}=$ $\tanh \left\{(T / L)\left[1 / \cos \left(\varphi_{\text {beam }}\right)\right] d \cos \left[(\pi / 2)+\varphi_{\text {beam }}+\theta^{\max }\right]\right\}$ (tanh is the hyperbolic tangent). As a result, $(T / L) d \approx$ 0.2294 . Finally, $\theta^{\max }\left(H_{x}\right)$ can be plotted as a function of $H_{x}$ for up and down domains [Fig. 5(a)]. First, at zero applied field, $\theta^{\max }$ equals $37^{\circ}$ and is close to the value obtained by the measurement at zero field with $\varphi_{\text {beam }}$ fixed to $30^{\circ}$.

Combining the measured $\theta^{\max }\left(H_{X}\right)$ presented in Fig. 5(a) to the spatial cosine variation of $\theta(y)$ in Fig. 4, we reconstruct the $M(H)$ loop with the field applied in plane [red dots in Fig. 5(b)]. We calculate the average, over a stripe period along $y$, projection of the magnetization along $x$ using

$M_{x}\left(H_{x}\right)=\frac{1}{T_{\text {stripe }}} \int_{0}^{T_{\text {stripe }}} \cos \left[\theta^{\max }\left(H_{x}\right) \cos \left(2 \pi \frac{y}{T_{\text {stripe }}}\right)\right] d y$,

where $T_{\text {stripe }}$ is the period of the stripes.

It appears clearly that the remanence in this reconstruction is overestimated [red dots in Fig. 5(b)]. As reported in previous studies [1], closure domains can exist with the magnetization component in plane and perpendicular to the applied field, which would reduce strongly the remanence. In our experimental STXM configuration, these closure domains cannot be observed, since their magnetization is mostly perpendicular to the x-ray beam. Rotating the sample $90^{\circ}$ to align the impinging $\mathrm{x}$-ray with respect to the $y$ direction does not allow the flux closure domain structure to be solved because the XMCD contrast is mainly dominated by the contribution of the out-of-plane component of magnetization. We use micromagnetic calculations to confirm that the low remanence is linked to the presence of closure domains, and this is done using Mumax3 [26]. All magnetic parameters of $\mathrm{Co}_{40} \mathrm{Fe}_{40} \mathrm{~B}_{20}$ are extracted from experiments; the incertitude on the value of anisotropy is the largest [27]. We can reproduce all characteristic parameters of the domain structure: the domain period [experiments: $315 \mathrm{~nm}$ from Fig. 2, simulations: $335 \mathrm{~nm}$ (a)
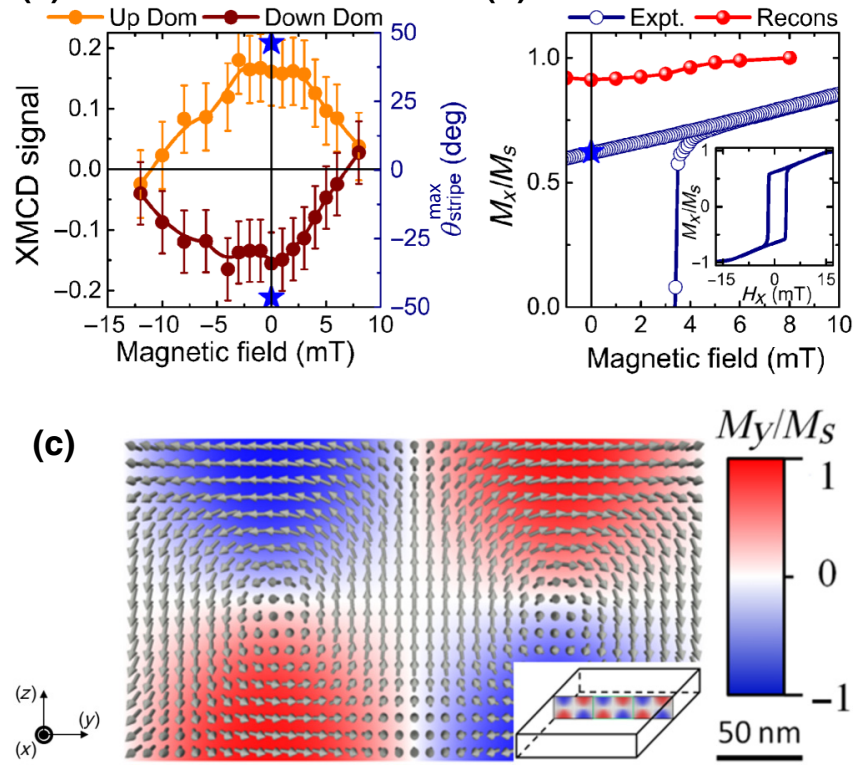

FIG. 5. (a) $<\mathrm{XMCD}^{\max }>$ versus the magnetic field, $H_{x}$, along the $x$ axis for up (orange) and down (brown) domains, and variation of the maximum stripe angle. Errors bars are the standard deviation of $\mathrm{XMCD}^{\max }$ on the same figure, variation of maximum stripe angle. (b) Magnification of a region of interest of $M_{x} / M_{s}$ versus $H_{x}$ recorded with a vibrating sample magnetometer (open blue circles). Inset is the total hysteresis cycle. Red dots are reconstructed values of $M_{x} / M_{s}$ from the XMCD signal. Blue star is the remanence calculated using micromagnetic calculations. (c) Magnetization map calculated using MuMax3 in the plane perpendicular to the stripes (Muview2 code is used to plot vectors). Displayed unit cell corresponds to one period (equal to $335 \mathrm{~nm}$ ) of the stripes and contains two walls; this cell is replicated by periodic boundary conditions into $x$ and $y$ to model the infinite film.

from Fig. 5(c)], saturation fields [experiments: $13 \mathrm{mT}$ from Fig. 5(b), inset; simulations: $13 \mathrm{mT}$ (not shown)], in-plane remanence \{experiments: 0.62 from Fig. 5(b), inset; simulations: 0.6225 [blue star in Fig. 5(b)]\}. As far as the stripe angle is concerned, the micromagnetic simulations show the existence of Néel caps that close the magnetic flux at the surface. At the center of the stripe, the magnetization angle varies from $73^{\circ}$ at the center of the film to $6^{\circ}$ at the surface. In the geometry of the last experiment, i.e., $\varphi_{\text {beam }}=0^{\circ}$, it is simple to show from Eq. (1) that $I=I_{0} e^{-\frac{T}{L}\left(1+d\left\langle m_{z}\right\rangle / m_{S}\right)}$, where $\left\langle m_{z}\right\rangle$ is the average of the $z$ component of magnetization over the film thickness. Using micromagnetic calculations, a mean value of stripe angle of $46.4^{\circ}$ can be calculated. It is slightly higher than the mean value determined experimentally, but within the measurement uncertainty. The difference arises from the difficulty in adjusting the value of the anisotropy (within the uncertainty of measurement) to reproduce all characteristic parameters of the domain structure [27], but also 
from averaging of the angle due to the size of the photon beam (36 $\mathrm{nm}$ in diameter).

\section{CONCLUSIONS}

By the use of high-resolution scanning transmission $\mathrm{x}$ ray microscopy, we have quantitative access to the mean canting angles relative to the surface normal and a sense of weak stripe domains. We extend previous reports to measure a single angle by the use of x-ray beam polarization. We are also able to gain access to the spatial variation of the stripe angle and measure the canting angle versus applied field. This method is applied to $180-\mathrm{nm}-$ thick $\mathrm{Co}_{40} \mathrm{Fe}_{40} \mathrm{~B}_{20}$ for which canting angles of between $33.9^{\circ}$ and $37^{\circ}$ can be measured. We shed light on the fact that, without a combination of techniques, one can miss out on the presence of Néel flux closure domains. This kind of study should be completed by a more surface- and in-plane-magnetization-component-sensitive microscopy technique, such as $\mathrm{x}$-ray photoemission electron microscopy to gain a complete understanding of the micromagnetic configuration.

Beyond characterization of the WS angle, this quantitative x-ray magnetic microscopy can be used to study the local textures hosted in all materials that exhibit some circular x-ray magnetic dichroism.

\section{ACKNOWLEDGMENTS}

The STXM measurements are done on the Hermes Beamline at Synchrotron SOLEIL. The authors acknowledge assistance from SOLEIL Hermes beamline staff. This work is partially supported by SOLEIL and the ANR SWANGATE. Sample growth and macroscopic measurements are performed using equipment from the TUBE Daum funded by FEDER (EU), ANR, the Region Lorraine, and Grand Nancy.

[1] A. Hubert and R. Schäfer, Magnetic Domains: the Analysis of Magnetic Microstructures (Springer Science \& Business Media, Springer-Verlag, Berlin, Heidelberg, 2008).

[2] A. V. Svalov, I. R. Aseguinolaza, A. Garcia-Arribas, I. Orue, J. M. Barandiaran, J. Alonso, M. L. FernandezGubieda, and G. V. Kurlyandskaya, Structure and magnetic properties of thin permalloy films near the "transcritical" state, IEEE Trans. Magn. 46, 333 (2010).

[3] N. R. Álvarez, J. E. Gómez, A. E. Moya Riffo, M. A. Vicente Álvarez, and A. Butera, Critical thickness for stripe domain formation in FePt thin films: Dependence on residual stress, J. Appl. Phys. 119, 083906 (2016).

[4] C. Blanco-Roldán, C. Quirós, A. Sorrentino, A. HierroRodríguez, L. Álvarez-Prado, R. Valcárcel, M. Duch, N. Torras, J. Esteve, J. I. Martín, M. Vélez, J. M. Alameda, E. Pereiro, and S. Ferrer, Nanoscale imaging of buried topological defects with quantitative X-ray magnetic microscopy, Nat. Commun. 6, 8196 (2015).

[5] A. V. Svalov, G. S. Kandaurova, and O. I. Bespalko, Rotating anisotropy in Gd-Co amorphous films, Fiz. Met. Metalloved. 80, 65 (1995).

[6] J. M. Guzmán, N. Álvarez, H. R. Salva, M. Vásquez Mansilla, J. Gómez, and A. Butera, Abnormal temperature dependence of the coercive field in FePt thin films, J. Magn. Magn. Mater. 347, 61 (2013).

[7] D. Wu, T. Jin, Y. Lou, and F. Wei, Understanding the dense stripe domains in soft magnetic film, Appl. Surf. Sci. 346, 567 (2015).

[8] C. B. Craus, A. R. Chezan, M. H. Siekman, J. C. Lodder, D. O. Boerma, and L. Niesen, Stripe domains in $\mathrm{Fe}-\mathrm{Zr}-\mathrm{N}$ nanocrystalline films, J. Magn. Magn. Mater. 240, 423 (2002).

[9] M. Di Pietro Martínez, J. Milano, M. Eddrief, M. Marangolo, and S. Bustingorry, Modeling magnetization curves in magnetic thin films with striped patterns, J. Phys.: Condens. Matter 28, 136001 (2016).

[10] M. Coïsson, F. Vinai, P. Tiberto, and F. Celegato, Magnetic properties of FeSiB thin films displaying stripe domains, J. Magn. Magn. Mater. 321, 806 (2009).

[11] Z. G. Sun, H. Kuramochi, M. Mizuguchi, F. Takano, Y. Semba, and H. Akinaga, Magnetic properties and domain structures of FeSiB thin films, Surf. Sci. 556, 33 (2004).

[12] W. Tee Soh, N. N. Phuoc, C. Y. Tan, and C. K. Ong, Magnetization dynamics in permalloy films with stripe domains, J. Appl. Phys. 114, 053908 (2013).

[13] J. McCord, B. Erkartal, T. von Hofe, L. Kienle, E. Quandt, O. Roshchupkina, and J. Grenzer, Revisiting magnetic stripe domains - anisotropy gradient and stripe asymmetry, J. Appl. Phys. 113, 073903 (2013).

[14] J. Ben Youssef, N. Vukadinovic, D. Billet, and M. Labrune, Thickness-dependent magnetic excitations in permalloy films with nonuniform magnetization, Phys. Rev. B 69, 174402 (2004).

[15] N. Amos, R. Fernandez, R. Ikkawi, B. Lee, A. Lavrenov, A. Krichevsky, D. Litvinov, and S. Khizroev, Magnetic force microscopy study of magnetic stripe domains in sputter deposited permalloy thin films, J. Appl. Phys. 103, 07 E732 (2008).

[16] P. Sharma, H. Kimura, A. Inoue, E. Arenholz, and J.-H. Guo, Temperature and thickness driven spin-reorientation transition in amorphous Co-Fe-Ta-B thin films, Phys. Rev. B 73, 052401 (2006).

[17] M. Hehn, S. Padovani, K. Ounadjela, and J. P. Bucher, Nanoscale magnetic domain structures in epitaxial cobalt films, Phys. Rev. B 54, 3428 (1996).

[18] A. K. Singh, B. Kisan, D. Mishra, and A. Perumal, Thickness dependent magnetic properties of amorphous FeTaC films, J. Appl. Phys. 111, 093915 (2012).

[19] C. Das and P. Alagarsamy, Tuning the magnetic properties of stripe domain structured $\mathrm{CoFeB}$ films using stack structure with spacer layer thickness dependent interlayer coupling, J. Magn. Magn. Mater. 448, 23 (2018).

[20] A. Gayen, G. K. Prasad, S. Mallik, S. Bedanta, and A. Perumal, Effects of composition, thickness and temperature on the magnetic properties of amorphous CoFeB thin films, J. Alloys Compd. 694, 823 (2017). 
[21] D. Garci'a, J. L. Muñoz, G. Kurlyandskaya, M. Vázquez, M. Ali, and M. R. J. Gibbs, Induced anisotropy, magnetic domain structure and magnetoimpedance effect in $\mathrm{CoFeB}$ amorphous thin films, J. Magn. Magn. Mater. 191, 339 (1999).

[22] Y. Henry, D. Stoeffler, J.-V. Kim, and M. Bailleul, Unidirectional spin-wave channeling along magnetic domain walls of Bloch type, Phys. Rev. B 100, 024416 (2019).

[23] T. Petrisor, Modulated magnetic structures for vortex pinning in high temperature superconductors, PhD Lorraine University, https://hal.univ-lorraine.fr/tel-01746237 (2018).

[24] R. Belkhou, S. Stanescu, S. Swaraj, A. Besson, M. Ledoux, M. Hajlaoui, and D. Dalle, HERMES: A soft X-ray beamline dedicated to X-ray microscopy, J. Synchrotron. Rad. 22, 968 (2015).

[25] T. Funk, A. Deb, S. J. George, H. Wang, and S. P. Cramer, $\mathrm{X}$-ray magnetic circular dichroism - a high energy probe of magnetic properties, Coord. Chem. Rev. 249, 3 (2005).

[26] A. Vansteenkiste, J. Leliaert, M. Dvornik, M. Helsen, F. Garcia-Sanchez, and B. Van Waeyenberge, The design and verification of $\mathrm{MuMax}_{3}$, AIP Adv. 4, 107133 (2014).

[27] $M_{s}=1327 \mathrm{kA} / \mathrm{m}$ (measured by SQUID), $A_{\mathrm{ex}}=17 \mathrm{pJ} / \mathrm{m}$ (measured by FMR), $K=36 \mathrm{~kJ} / \mathrm{m}^{3}$ uniaxial perpendicular to film plane (measured by FMR and SQUID), cell size $1.3 \times 1.4 \times 3 \mathrm{~nm}^{3}$. K. Ait Oukaci, D. Stoeffler, M. Grassi, B. Sarpi, M. Bailleul, Y. Henri, F. Montaigne, R. Belkhou, M. Hehn, D. Lacour, in preparation (2020). 